\title{
Pancreatic hamartoma, a rare benign disease of the pancreas: A case report
}

\author{
JIANWEI ZHANG, HUI WANG, XIAOLONG TANG, QINGLONG JIANG and CHENGFENG WANG \\ Department of Abdominal Surgery, Chinese Academy of Medical Sciences, Cancer Hospital, \\ Beijing 100021, P.R. China
}

Received January 3, 2015; Accepted February 8, 2016

DOI: $10.3892 / \mathrm{ol} .2016 .4535$

\begin{abstract}
Pancreatic hamartoma is an extremely rare, non-neoplastic, mass-forming lesion that may be mistaken for malignancy, and the pre-operative diagnosis is particularly challenging. The published literature contains only 23 cases of pancreatic hamartoma. The majority of the cases reported patients with a single benign tumor-like disease that received a pancreatectomy. Immunohistochemical findings confirmed the diagnosis following surgery. The current study reports the case of a 53-year-old female who presented to the Department of Abdominal Surgery, Chinese Academy of Medical Sciences, Cancer Hospital (Beijing, China), due to abdominal pain. Abdominal magnetic resonance imaging revealed a $22 \times 14-m m$ mass in the head of the pancreas. The patient was pre-operatively diagnosed with a pancreatic space-occupying lesion, and subsequently underwent a pancreaticoduodenectomy. The post-operative course was uneventful. Histological examination of the resected lesion resulted in a diagnosis of pancreatic hamartoma. There were no signs of recurrence at 55 months post-surgery.
\end{abstract}

\section{Introduction}

Hamartomas are benign tumors formed from the disordered growth of normal cells and tissues at the affected site (1). Pancreatic hamartoma is extremely rare and accounts for $<1 \%$ of hamartoma; the etiology is unknown and the disease has a low morbidity (2). With the increased utilization of imaging technology, such as endoscopic ultrasound, there has been an increased detection of asymptomatic lesions of the pancreas; however, the differentiation between hamartomas and other benign tumors or malignancies is extremely difficult (3). The final diagnosis is usually made according to histopathological and immunohistochemical results (4).

Correspondence to: Dr Chengfeng Wang, Department of Abdominal Surgery, Chinese Academy of Medical Sciences, Cancer Hospital, 17 Panjiayuan, Chaoyang, Beijing 100021, P.R. China E-mail: panchutong@163.com

Key words: pancreas, hamartoma, surgery
Due to the dangers of biliary obstruction or hemorrhage, a pancreatectomy is necessary (5). No recurrence usually occurs following radical excision. Pancreatic hamartoma can be divided into two subgroups: Solid and cystic, or solid (6). The majority of cases reported in the literature are cystic hamartomas (1-3,7-13). The present study reports the case of a solid pancreatic hamartoma that was diagnosed after surgery.

\section{Case report}

A 53-year old female was admitted to the Department of Abdominal Surgery, Chinese Academy of Medical Sciences, Cancer Hospital (Beijing, China) in October 2014, due to a 2-month history of abdominal pain, accompanied by slight anorexia and $2 \mathrm{~kg}$ of weight loss. Physical examination did not detect any abnormalities. The history of the patient did not disclose any systemic diseases or previous surgeries. The patient reported no alcohol consumption or cigarette use, and there was no family history of tumor-associated disease. Blood tests indicated that tumor marker levels [carcinoembryonic antigen, carbohydrate antigen (CA)19-9, CA-125 and CA72-4] were within the normal ranges, and pancreatic exocrine and endocrine function was sufficient. Other standard blood tests were unremarkable. Abdominal magnetic resonance imaging (MRI; MAGNETOM Skyra, Siemens Healthcare, Erlangen, Germany) was performed and revealed a 22x14-mm, predominantly well-circumscribed lesion in the pancreatic tuberculosis. The lesion exhibited a low signal on T1-weighted images (T1WI) and T2WI/fat suppression images, accompanied by certain hyperintense areas (Fig. 1). Endosonography (TGF-UC260J; Olympus Corporation, Tokyo, Japan) was also performed and revealed a $2.83 \times 2.27-\mathrm{cm}$, hypoechogenic lesion located in the head of the pancreas. Endoscopic ultrasound (EUS) fine-needle aspiration was not performed due to the problematic position of the lesion, which was particularly close to the superior mesenteric vein (Fig. 2). The combined imaging results were not enough to form a reliable diagnosis; therefore, a formal pancreaticoduodenectomy was performed. During the surgery, a $2.3 \times 1.5 \times 1.5-\mathrm{cm}$, firm mass with an intact capsule was identified, which appeared to be embedded in the parenchyma of the head of the pancreas (Fig. 3). On the resected surface of the tumor, a well-demarcated, 


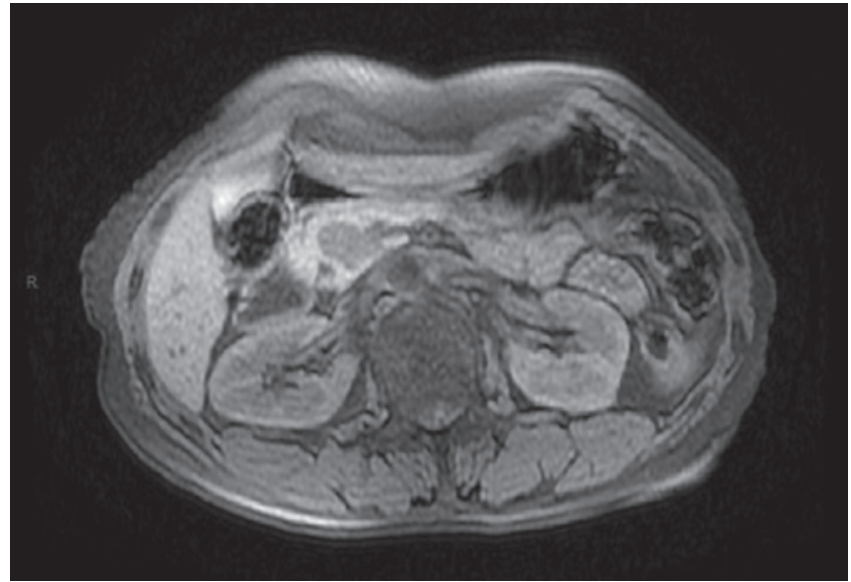

Figure 1. Magnetic resonance imaging showing a well-circumscribed, 22-mm lesion of the head of the pancreas, with a low signal on T1-weighted imaging.

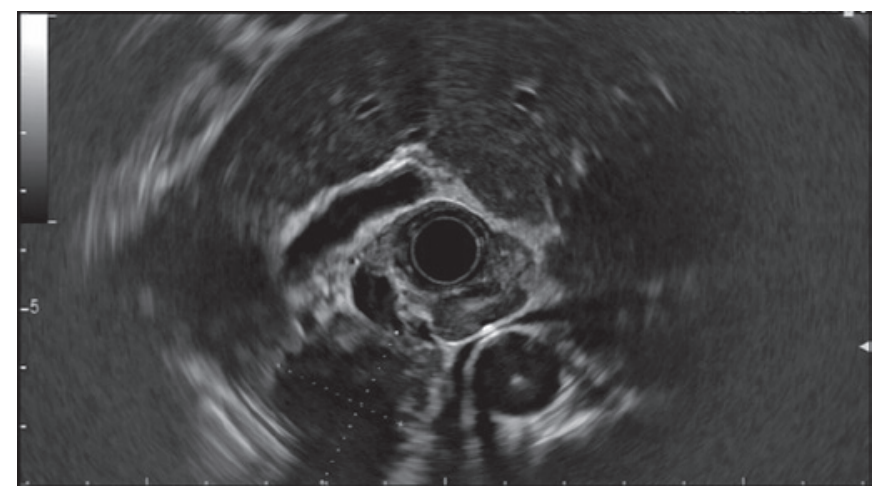

Figure 2. Endosonography image revealing a $2.83 \times 2.27-\mathrm{cm}$, hypoechogenic lesion located in the head of the pancreas, close to the superior mesenteric vein.

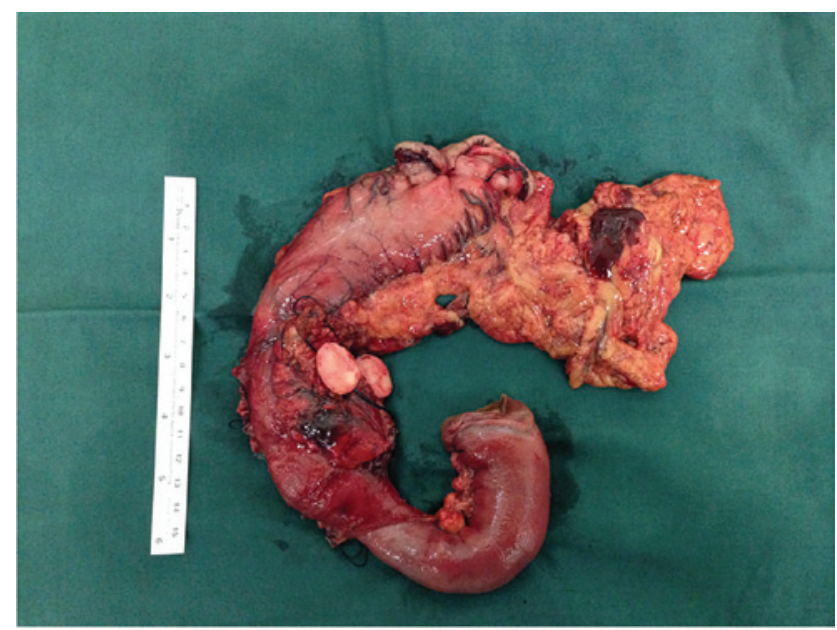

Figure 3. Tumor embedded in the parenchyma of the head of the pancreas, with a white resected surface and intact capsule.

solid, homogeneously white-colored nodule was identified. The resected tissue was formalin (Beijing Saichi Biological Technology Co., Ltd., Beijing, China)-fixed, paraffin (Beijing Saichi Biological Technology Co., Ltd., Beijing, China)-embedded and cut into $4-\mu \mathrm{m}$ sections prior to microscopic examination (CX23; Olympus Corporation),

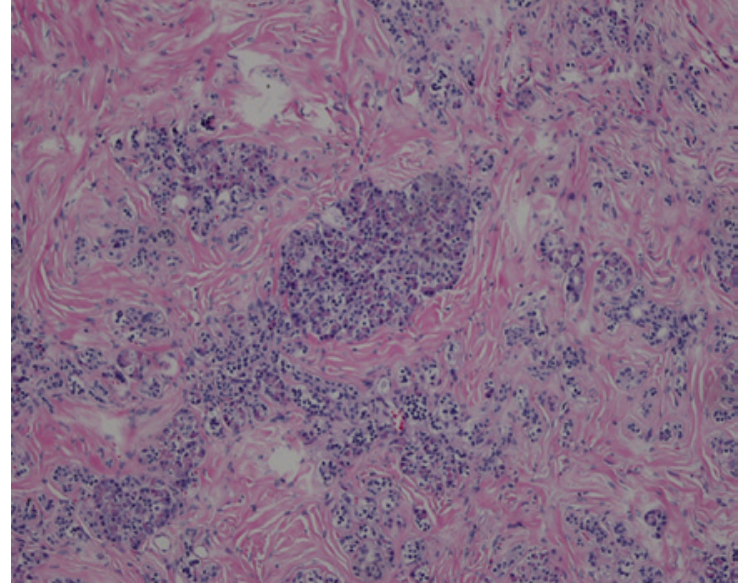

Figure 4. Microscopic analysis of the resected tissue. The lesion was composed of disarranged pancreatic acinar and ductal cells, embedded in a markedly fibrous stroma. Hematoxylin-eosin staining; magnification, x100.

which demonstrated that the lesion was composed of disarranged ductal and acinar cells, embedded in a markedly fibrous stroma (hematoxylin and eosin stain; Beijing Saichi Biological Technology Co., Ltd.) (Fig. 4). Immunohistochemical staining indicated that the lesion was positive for $\alpha-1$-antitrypsin, $\alpha-1$-antichymotrypsin and s100, with a Ki-67 score of $<1 \%$. Small amounts of disordered acinar cells and ductal epithelium were immunohistochemically positive for cluster of differentiation (CD)117, CD56, chromogranin A, progesterone receptor and synaptophysin, and negative for cytokeratin 19, p63 and vimentin. Thus, the histological diagnosis was confirmed as pancreatic hamartoma. The post-operative clinical course was stable without complications, and the patient was discharged following 15 days of hospitalization. At 55 months of follow-up, no recurrence was observed.

\section{Discussion}

A hamartoma is a focal, benign malformation that resembles a neoplasm in the tissue of its origin (14). This lesion is not a real tumor, and it grows at the same rate as its adjacent tissues. Hamartomas may develop in various areas of the body, and are typically observed in the lungs, heart, kidneys, spleen or other vascular organs. These lesions are commonly asymptomatic and may remain undetected unless identified incidentally during imaging analyses (14).

Pancreatic hamartoma is extremely rare. Only 23 cases, including the present case, have been reported in the literature (Table I), with the first case being described by Anthony et al in 1977 (7). The development of this disease may occur at any age, even in young children, however, the average age of occurrence is 40-60 years (median, 42.23 years) $(4,5$, $8-10,15-18)$. Morbidity is not equal between men and women, with a male to female ratio of 1.5:1. In contrast to hamartoma in other organs, the majority of pancreatic hamartoma cases are accompanied by pain, abdominal discomfort or certain vague symptoms, including dyspepsia and weight loss $(11,19)$. Tumors are often located in the head of the pancreas, but signs of jaundice are rare. 


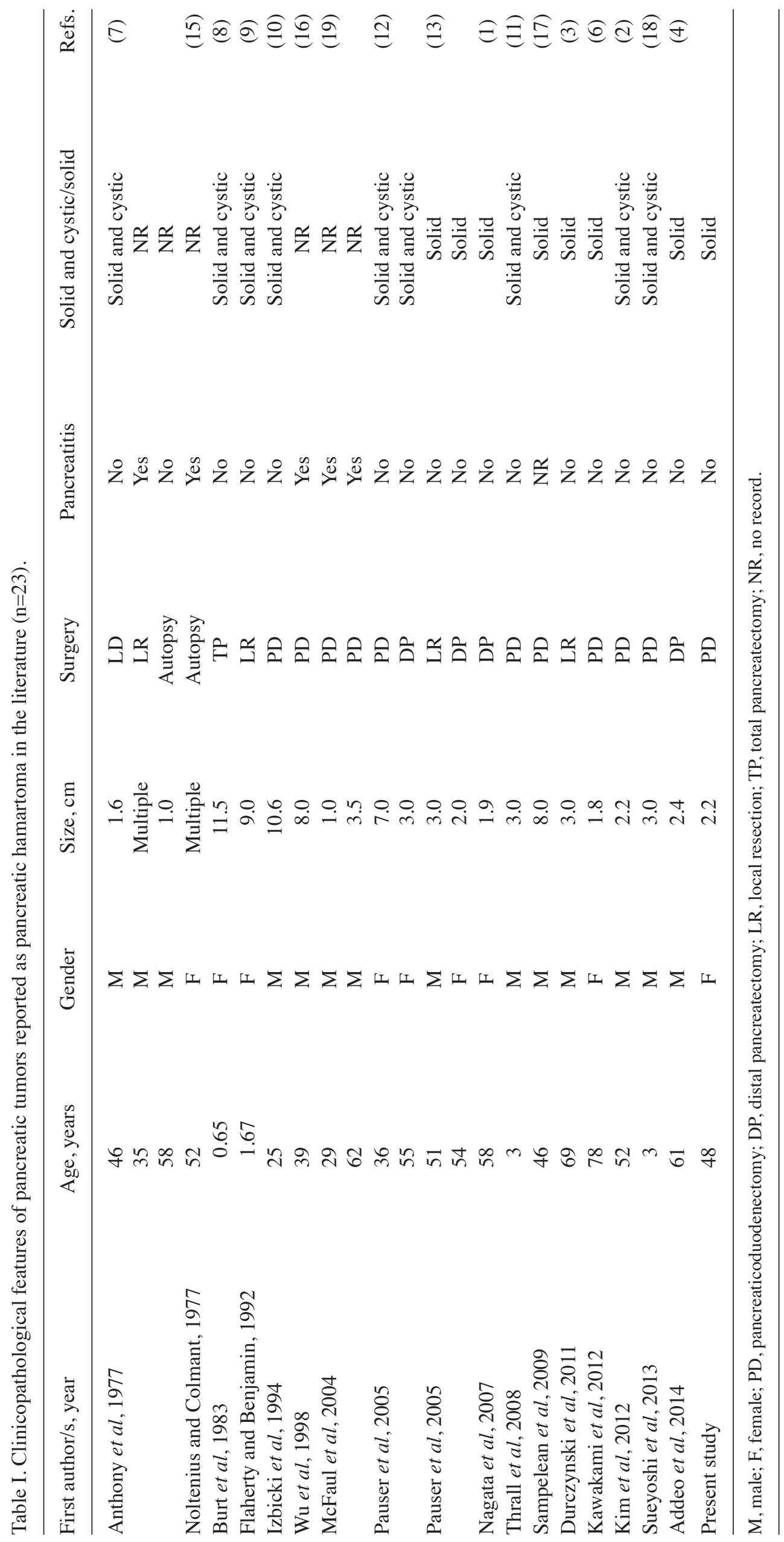


Pancreatic hamartoma is typically composed of disorganized, well-differentiated exocrine and endocrine pancreatic tissue. Acinar, islet and ductal cells are the three main components that form the lesions (1). A specific cell type, termed spindle-shaped cells, exhibit immunoreactivity for CD34 and CD117 in a number of pancreatic hamartoma cases $(1,12)$. Pancreatic hamartoma is classified as solid or solid and cystic by macroscopic findings (13). Although the pathogenesis and origin of this disease remains unclear, it should only be diagnosed in patients without evidence of chronic pancreatitis, as chronic pancreatitis often presents with a depletion of acinar cells in the fibrous stroma, thus mimicking hamartoma with a lack of such cells (12).

To date, the existing imaging methods employed to characterize pancreatic hamartoma lack the accuracy to successfully distinguish it from other diseases. The majority of lesions that develop in the pancreas are malignant or have malignant potential, therefore, surgery is the optimal treatment in such instances, and a complete resection with negative margins is imperative (3). The treatment of choice for hamartoma is a conventional pancreatectomy or a pancreas-preserving surgery (central pancreatectomy or enucleation). A conventional pancreatectomy, including pancreatoduodenectomy and distal pancreatectomy, may sacrifice the normal pancreatic parenchyma and be associated with the risk of post-operative diabetes mellitus or endocrine and exocrine pancreatic insufficiency (2). Considering the benign behavior of this tumor, pancreas-preserving surgery is recommended, which has the advantages of preserving the integrity of the gastrointestinal tract and splenic function, and sparing the maximal pancreatic endocrine and exocrine function by avoiding an extended resection of the pancreas (20). However, there is debate regarding the use of this treatment. Since the majority of pancreatic hamartoma cases have a good prognosis and pancreatectomies are prone to the development of fistulas or other complications, the 'wait and see' policy is an alternative treatment for asymptomatic patients in order to exclude the diagnosis of a malignant tumor (20).

In conclusion, pancreatic hamartoma may be detected incidentally, without the patient presenting with any signs or symptoms of disease. Clinical symptoms are dependent on tumor size and location, and the diagnosis of this disease primarily depends on imaging techniques, including computed tomography, MRI and EUS. However, pancreatic hamartoma is difficult to differentiate from other benign pancreatic lesions. For symptomatic patients or those with an indefinite diagnosis, a complete surgical excision is recommended. Following a successful pancreaticoduodenectomy, the patient in the present study was diagnosed with a pancreatic hamartoma on histological examination, and no recurrence has since been observed.

\section{References}

1. Nagata S, Yamaguchi K, Inoue T, Yamaguchi H, Ito T, Gibo J, Tanaka M and Tsuneyoshi M: Solid pancreatic hamartoma. Pathol Int 57: 276-280, 2007.

2. Kim HH, Cho CK, Hur YH, Koh YS, Kim JC, Kim HJ, Kim JW, Kim Y and Lee JH: Pancreatic hamartoma diagnosed after surgical resection. J Korean Surg Soc 83: 330-334, 2012.

3. Durczynski A, Wiszniewski M, Olejniczak W, Polkowski M, Sporny S and Strzelczyk J: Asymptomatic solid pancreatic hamartoma. Arch Med Sci 7: 1082-1084, 2011.

4. Addeo P, Tudor G, Oussoultzoglou E, Averous G and Bachellier P: Pancreatic hamartoma. Surgery 156: 1284-1285, 2014.

5. Inoue $\mathrm{H}$, Tameda M, Yamada R, Tano S, Kasturahara M, Hamada Y, Tanaka K, Horiki N andTakei Y: Pancreatic hamartoma: A rare cause of obstructive jaundice. Endoscopy 46 (Suppl 1): 157-158, 2014.

6. Kawakami F, Shimizu M, Yamaguchi H, Hara S, Matsumoto I, $\mathrm{Ku}$ Y and Itoh T: Multiple solid pancreatic hamartomas: A case report and review of the literature. World J Gastrointest Oncol 4: 202-206, 2012.

7. Anthony PP, Faber RG and Russell RC: Pseudotumours of the pancreas. BMJ 1: 814, 1977.

8. Burt TB, Condon VR and Matlak ME: Fetal pancreatic hamartoma. Pediatr Radiol 13: 287-289, 1983.

9. Flaherty MJ and Benjamin DR: Multicystic pancreatic hamartoma: A distinctive lesion with immunohistochemical and ultrastructural study. Hum Pathol 23: 1309-1312, 1992.

10. Izbicki JR, Knoefel WT, Müller-Höcker J and Mandelkow HK: Pancreatic hamartoma: A benign tumor of the pancreas. Am J Gastroenterol 89: 1261-1262, 1994.

11. Thrall M, Jessurun J, Stelow EB, Adsay NV, Vickers SM, Whitson AK, Saltzman DA and Pambuccian SE: Multicystic adenomatoid hamartoma of the pancreas: A hitherto undescribed pancreatic tumor occurring in a 3-year-old boy. Pediatr Dev Pathol 11: 314-320, 2008.

12. Pauser U, da Silva MT, Placke J, Klimstra DS and Klöppel G: Cellular hamartoma resembling gastrointestinal stromal tumor: A solid tumor of the pancreas expressing c-kit (CD117). Mod Pathol 18: 1211-1216, 2005.

13. Pauser U, Kosmahl M, Kruslin B, Klimstra DS and Klöppel G: Pancreatic solid and cystic hamartoma in adults: Characterization of a new tumorous lesion. Am J Surg Pathol 29: 797-800, 2005.

14. Heald B, Burke CA, Kalady M and Eng C: ACG guidelines on management of PTEN-hamartoma tumor Ssyndrome: Does the evidence support so much so young? Am J Gastroenterol 110: $1733-1734,2015$.

15. Noltenius $\mathrm{H}$ and Colmant HJ: Excessive hyperplasia of the exocrine pancreatic tissue and Wernicke's encephalopathy (author's transl). Med Klin 72: 2155-2158, 1977 (In German).

16. Wu SS, Vargas HI and French SW: Pancreatic hamartoma with Langerhans cell histiocytosis in a draining lymph node. Histopathology 33: 485-487, 1998.

17. Sampelean D, Adam M, Muntean V, Hanescu B and Domsa I: Pancreatic hamartoma and SAPHO syndrome: A case report. J Gastrointestin Liver Dis 18: 483-486, 2009.

18. Sueyoshi R, Okazaki T, Lane GJ, Arakawa A, Yao T and Yamataka A: Multicystic adenomatoid pancreatic hamartoma in a child: Case report and literature review. Int J Surg Case Rep 4: 98-100, 2013.

19. McFaul CD, Vitone LJ, Campbell F, Azadeh B, Hughes ML, Garvey CJ, Ghaneh P, Neoptolemos JP and Longnecker DS: Pancreatic hamartoma. Pancreatology 4: 533-538, 2004.

20. Beger HG, Siech M, Poch B, Mayer B and Schoenberg MH: Limited surgery for benign tumours of the pancreas: A systematic review. World J Surg 39: 1557-1566, 2015. 